\title{
Notas Ecológicas Preliminares sobre Macro-fauna Bentónica y su Relación con Aves Playeras
}

\section{Ecological Notes on the Interaction between Macrobenthos and Shore birds frontier: A Preliminary Study}

INFORMACIÓN DEL ARTÍCULO

Fecha de recepción: 11 de Febrero de 2018.

Fecha de aceptación: 02 de Julio de 2018.

\footnotetext{
1 Grupo de Toxicología Ambiental, Facultad Ciencias de la Vida, Escuela Superior Politécnica del Litoral (ESPOL). Campus Gustavo Galindo, $\mathrm{Km} 30.5$ Vía Perimetral, P.O.Box 09-01-5863, Guayaquil.
}

2 Laboratorio de Zoología, Facultad Ciencias de la Vida, Escuela Superior Politécnica del Litoral (ESPOL). Campus Gustavo Galindo, Km 30.5 Vía Perimetral, P.O.Box 09-01-5863, Guayaquil.

Autor de correspondencia: Antonio Herrera, Facultad Ciencias de la Vida, Escuela Superior Politécnica del Litoral (ESPOL). Campus Gustavo Galindo, Km 30,5 Vía Perimetral, P.O.Box 09-01-5863, Guayaquil- Ecuador.

E-mail: anheni@espol.edu.ec

Tlf: (593-4) 226-9610

ENLACE DOI:

http://10.31095/investigatio.2018.11.5

Antonio Herrera $^{1 *}$, Paolo Piedrahita $^{2}$, Paola Calle $^{1}$

\section{Resumen}

En Ecuador se han realizado investigaciones sobre la macrofauna asociada a playas arenosas, pero no se ha estudiado la relación de esta macrofauna con aves playeras. Se tiene como propósito mostrar hallazgos preliminares en cuanto a la relación ecológica entre los macroinvertebrados bentónicos y las aves playeras en la playa El Pelado, Provincia del Guayas, Ecuador. Se establecieron dos transectos perpendiculares a la línea de costa sobre la zona intermareal, divididos en tres zonas. En cada zona se tomaron muestras para evaluar la macrofauna. El monitoreo de la avifauna se efectuó mediante observaciones directa hacia la zona intermareal, con el fin de identificar a las especies que muestren comportamientos de alimentación. La macrofauna presenta un patrón espacial relacionado con las zonas evaluadas, en donde los poliquetos de la familia Opheliidae y el gastrópodo Olivella semistriata presentaron altas densidades $\left(>700 \mathrm{ind} / \mathrm{m}^{2}\right)$. Las especies de aves observadas (Haematopus palliatus, Charadrius vociferus, Numenius phaeopus, y Tringa incana) introdujeron activamente sus picos en la arena, indicio comportamental de forrajeo de macrofauna bentónica.

Palabras Clave:

Avifauna, Charadrius vociferus, Ecuador, Haematopus palliatus, Numenius phaeopus, Olivella semistriata, playa arenosa, Tringa incana.

Clasificación JEL: Q29.

\begin{abstract}
Macrofauna studies in Ecuador have focused on species diversity and on population abundance of these organisms in sandy beaches. However, little is known about the relationship between microbenthic organism diversity and the foraging behavior of shore birds. Therefore, the aim of this work is to find out how macrobenthic diversity in sandy beaches relates to shore bird feeding behavior. This study was done in the sandy beach of El Pelado, Province of Guayas, Ecuador. We set two transects perpendicular to both the intertidal and the coastline zones. These transects were divided into three zones where samples were taken in order to evaluate macrofaunal diversity. On the other hand, shorebird surveys were done along the intertidal zone. We used binoculars and field guides to identify bird species when they were foraging. Our data showed a spatial pattern of macrofauna diversity related to the described zones, where polychaetes from the Opheliidae family and the gastropod Olivella semistriata showed the highest densities $\left(>700 \mathrm{ind} / \mathrm{m}^{2}\right)$. This macrofauna diversity was associated to the shore bird species we observed (Haematopus palliatus, Charadrius vociferus, Numenius phaeopus and Tringa incana) since all shore birds were actively foraging by introducing their beaks into the sandy soil.
\end{abstract}

Keywords:

Birdlife, Charadrius vociferus, Ecuador, Haematopus palliatus, Numenius phaeopus, Olivella semistriata, sandy beach, Tringa incana.

JEL Classification: Q29. 


\section{Introducción}

En el litoral oceánico ecuatoriano se han realizado levantamientos de información de la presencia y abundancia de organismos pertenecientes a comunidades de macrofauna bentónica, con especial énfasis en playas arenosas (Aerst aet al., 2004; Domínguez-Granda et al., 2004; Vanagt, 2007; Villamar \& Cruz, 2007; Villamar, 2009; Villamar, 2013; Marín-Jarrín et al., 2015; Marín-Jarrín et al., 2017). Sin embargo, estos estudios adolecen en cuanto a caracterizar los patrones de distribución tanto espaciales como temporales de dichas especies macrobentónicas, y el cómo estas comunidades se relacionan con parámetros ambientales del agua, sedimento y del morfodinamismo de la playa como reflejo de la energía del oleaje. Adicionalmente, no se encontraron artículos científicos sobre la interacción ecológica entre la comunidad del macrobento y su interacción (depredación) con aves playeras, quienes utilizan a las comunidades del macrozoobentos como recurso alimenticio. En Ecuador, los estudios de avifauna asociadas a los litorales intermareales se han enfocado en el levantamiento de información para inferir patrones migratorios y de biodiversidad (Haase, 2011; Agreda et al.,2013). Se conoce, de la literatura internacional, que parte de la dieta de las aves playeras se compone de invertebrados asociados a ecosistemas de las zonas intermareales (Hernández et al., 2010; Musmeci et al., 2013; Yan Yang et al., 2016).

Por lo expuesto anteriormente, existe la necesidad de estudios enfocados en examinar la interacción ecológica entre las comunidades del macrozoobentos como fuente de alimentos de aves playeras, sean estas especies migratorias o residentes. Este artículo tiene como propósito mostrar hallazgos preliminares en cuanto a la relación ecológica entre los macroinvertebrados bentónicos y las aves playeras en la playa El Pelado, Provincia del Guayas, Ecuador. Para ello se cumplirán los siguientes objetivos: caracterizar el ambiente del litoral intermareal mediante el estudio de la temperatura y salinidad en la columna de agua y del sedimento, estimar la pendiente de la playa y variables relacionadas al oleaje; y examinar la estructura comunitaria del macrozoobentos y la composición de especies de aves playeras asociado al litoral intermareal oceánico.

\section{Materiales y métodos}

\section{Área de estudio}

Playa El Pelado se encuentra ubicada en el perfil costanero ecuatoriano, tiene una extensión costera de $6 \mathrm{~km}$ hasta la población de Engabao, ubicado al suroeste de la provincia del Guayas, a 97 kilómetros de la ciudad de Guayaquil. El área de estudio está caracterizada por un clima tropical seco, con temperatura anual promedio entre $23^{\circ} \mathrm{C}$ y $25^{\circ} \mathrm{C}$, y precipitación anual inferior a $500 \mathrm{~mm}$, siendo el mes de marzo el más lluvioso (Nuques, 2012). Dicho perfil costero está compuesto principalmente por playas con fondo arenoso modulado por el oleaje, y por una plataforma rocosa ubicada al sur de dicha costa (Figura 1). 


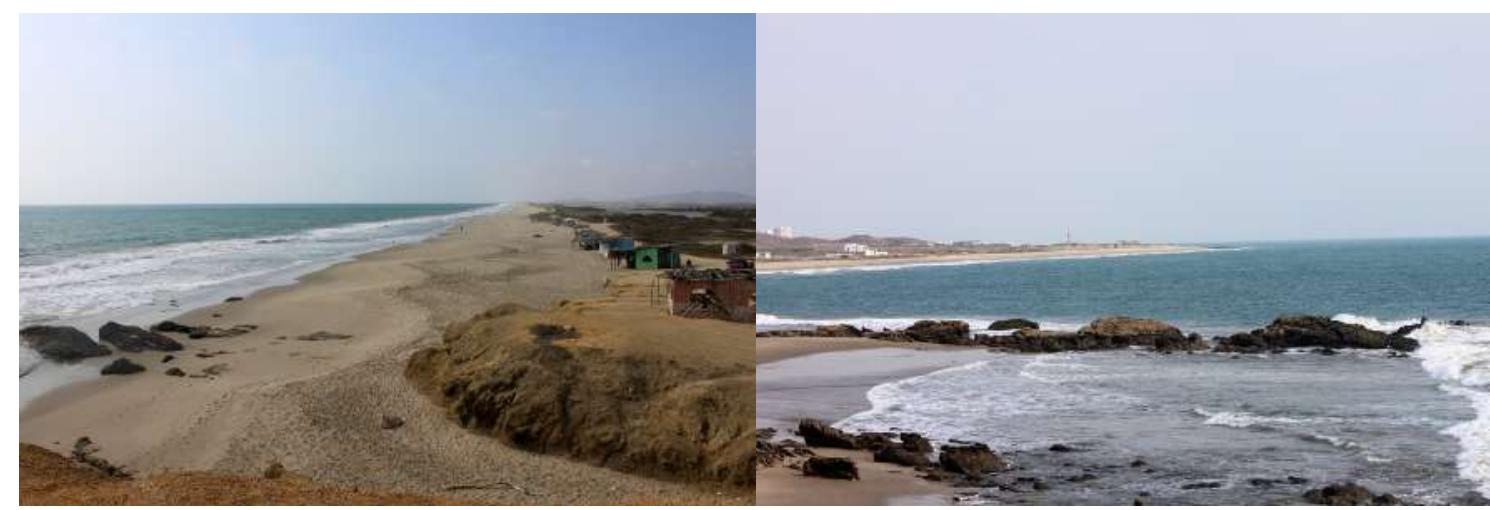

Figura 1. Fotografías del área de estudio, en donde se observa la playa arenosa y las plataformas rocosas evaluadas.

\section{Diseño del muestreo}

Se establecieron dos transectos perpendiculares a la línea de costa (Transecto 1: $2^{\circ} 37^{\prime} 3,5$ ' $\mathrm{S}, 80^{\circ} 27^{\prime}$ ' 3,5” $\mathrm{O}$; Transecto 2: $2^{\circ} 37^{\prime} 48,1^{\prime \prime} \mathrm{S}, 80^{\circ} 26^{\prime}$ 52,3” O), sobre la zona intermareal. Cada transecto se dividió en tres zonas correspondientes a la zona seca (supramareal), de retención (mesomareal), de resurgencia (mesomareal), basado en la propuesta de zonificación de Salvat (1964). El muestreo se realizó el 29 de julio de 2017, en el marco de la asignatura Zoología de Invertebrados de la carrera de Biología de la Escuela Superior Politécnica del Litoral.

\section{Caracterización ambiental}

Para la medición de la pendiente de la playa, se modificó la metodología propuesta por Emery (1961), mediante la utilización de un trípode con una cámara fotográfica ubicada al principio de la zona intermareal y una estaca colocada en la zona de lavado del oleaje, para coincidir la línea del horizonte con la estaca a través del lente y medir la altura hasta ese punto mediante una cinta métrica, y la distancia desde la berma hasta la estaca, lo cual se denominó como amplitud de la zona intermareal (Herrera, 2007). Para el cálculo de la pendiente se utilizó la siguiente ecuación:

$M=\frac{a}{L} x 100 ; \quad($ Pino \& Jaramillo, 1992)

donde " $a$ " es la altura medida en la estaca, y " $L$ " es la amplitud de la zona intermareal.

Se estimó la frecuencia del oleaje en términos de olas por minuto, calculando así el periodo medido en segundos. Con la ayuda de un termómetro y un refractómetro, se midió la temperatura en la arena, y la salinidad en una muestra de agua.

Recolección de muestras de arena para la caracterización de la comunidad macrobentónica

Para la recolección de muestras biológicas de macrofauna se utilizó un cilindro con $10 \mathrm{~cm}$ de diámetro. Para las tres zonas delimitadas en cada transecto se recolectaron tres muestras de arena, con un total de nueve muestras por cada 
transecto. Estas muestras fueron cernidas mediante un tamiz de $500 \mu \mathrm{m}, \mathrm{y}$ preservadas con formaldehido al $10 \%$ en solución con Eosina para su tinción. En el laboratorio se lavaron las muestras de arena con abundante agua para eliminar el formol en un tamiz de $500 \mu \mathrm{m}$, y mediante un estereoscopio con lámpara, se procedió a separar minuciosamente los organismos del material particulado. Luego se precedió a cuantificar e identificar hasta la jerarquía taxonómica más baja posible, con ayuda de guías fotográficas, y claves taxonómicas.

Análisis de datos de la comunidad macrobentónica

Se realizó un análisis multivariado permutacional de varianza (PERMANOVA, acrónimo en inglés; Anderson et al., 2008) para evaluar el comportamiento de los datos y determinar diferencias significativas, utilizando como fuentes de variación el transecto y la zona. Se realizaron las permutaciones de residuales bajo un modelo reducido con base en datos de abundancia de especies, transformados a la raíz cuarta (Clarke, 1993) y se consideró el índice de similitud de Bray Curtis para la estimación de las sumas cuadráticas. En forma complementaria, se realizó un Análisis de clasificación Jerárquica de Doble Vía (McCune et al.,
2002), para representar en forma diagramática la zonificación de las distintas especies de macroinvertebrados bentónicos.

\section{Registro de aves playeras en la zona intermareal}

El monitoreo de la avifauna se efectuó mediante observaciones directa hacia la zona intermareal con el fin de identificar a las especies que muestren comportamientos de alimentación en las zonas seca, de retención y de resurgencia en una playa arenosa.

Las observaciones de aves se realizaron el mismo día del muestreo de la macrofauna bentónica, es decir, durante la mañana, por un total de tres horas de monitoreo continuo. Para realizar estas observaciones, se dividió una longitud $1500 \mathrm{~m}$ de playa en cinco secciones iguales, las cuales fueron monitoreadas simultáneamente. Se utilizaron guías de campo para la identificación de las especies de aves, y binoculares de $8 \times 50 \mathrm{~mm}$.

\section{Resultados}

\section{Variables ambientales}

Se presentó un ambiente litoral con características oceánicas. Se presentan

Tabla 1.

Variables ambientales en playa El Pelado.

\begin{tabular}{ccccccc}
\hline Transecto & $\begin{array}{c}\text { Frecuencia } \\
\text { Oleaje } \\
\text { (olas/min) }\end{array}$ & $\begin{array}{c}\text { Período } \\
\text { Ola }(\mathrm{S})\end{array}$ & $\begin{array}{c}\text { Pendiente } \\
(\%)\end{array}$ & $\begin{array}{c}\text { Amplitud } \\
\text { Intermareal } \\
(\mathrm{m})\end{array}$ & $\begin{array}{c}\text { Temperatura } \\
\left({ }^{\circ} \mathrm{C}\right)\end{array}$ & $\begin{array}{c}\text { Salinidad } \\
(\text { UPS })\end{array}$ \\
\hline 1 & 8 & 7,5 & 7,03 & 32,7 & 26 & 35 \\
2 & 10 & 6 & 14,72 & 33,7 & 21 & 35 \\
\hline
\end{tabular}

60

INVESTIGATIO No. 11, noviembre 2018 Edición Especial, pp. 57-66,

ISSN: 1390 - 6399・ISSN-e: 2602 - 8336 


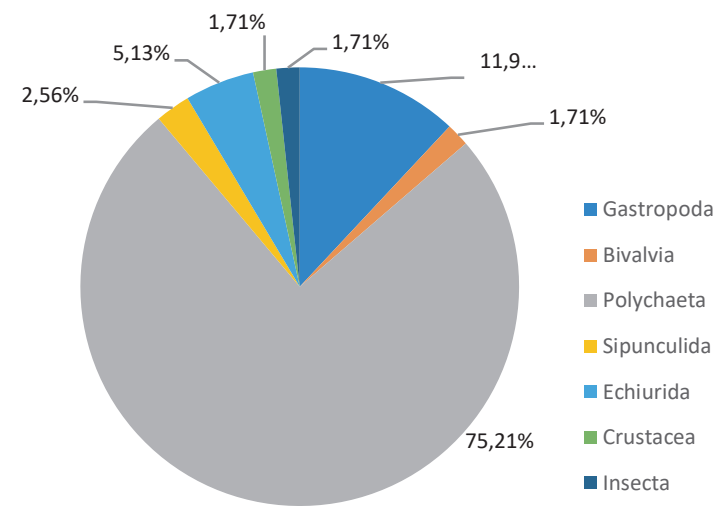

Figura 2. Porcentaje de abundancia de las distintas taxa en macrozoobentos de la playa El Pelado.

temperaturas entre 21 y $26^{\circ} \mathrm{C}$, con valores de salinidad de 35 ups (Tabla 1). En cuanto a las variables morfodinámicas, El Pelado es una playa con pendiente suave, entre 7,03\% (Transecto 1) y 14,72\% (Transecto 2), con frecuencia de oleaje que oscila entre 8 a 10 olas $/ \mathrm{min}$, con períodos de 6 a $7,5 \mathrm{~s}$.

Con relación al macrozoobentos, se identificaron ocho taxa, pertenecientes a

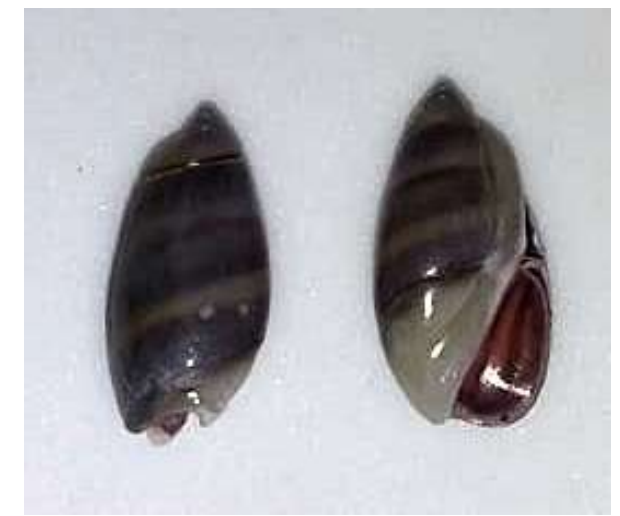

Figura 3. Ejemplares de Olivella semistriata. Se ha estimado que la longitud de este gasterópodo en su edad adulta en playas ecuatorianas está en el intervalo de 4 a $10 \mathrm{~mm}$ (Vanagt, 2007).

los siguientes grupos taxonómicos: Gasterópodos, Poliquetos, Sipuncúlidos, Echiuridos, Crustáceos e Insectos. Los poliquetos $(75,21 \%)$ presentaron el mayor porcentaje de abundancia, seguido por los moluscos gastrópodos $(11,97 \%)$ (Figura 2). En los poliquetos, la familia Opheliidae fue la más abundante (2600 ind $/ \mathrm{m}^{2}$, zona de retención), seguido por Spionidae (1250 ind $/ \mathrm{m}^{2}$, zona de resurgencia) (Tabla 2). En cuanto a los

\section{Tabla 2.}

Abundancia medida como densidad (ind $/ \mathrm{m}^{2}$ ), de los distintos grupos taxonómicos en el macrozoobentos de la Playa El Pelado.

\begin{tabular}{|c|c|c|c|c|c|c|}
\hline \multirow[b]{2}{*}{ Taxón } & \multicolumn{3}{|c|}{ Transecto 1} & \multicolumn{3}{|c|}{ Transecto 2} \\
\hline & Seca & Retención & Resurgencia & Seca & Retención & Resurgencia \\
\hline \multicolumn{7}{|l|}{ Gastropoda } \\
\hline Olivella semistriata & 0 & 700 & 0 & 0 & 0 & 0 \\
\hline \multicolumn{7}{|l|}{ Bivalvia } \\
\hline Donax mancorensis & 0 & 50 & 50 & 0 & 0 & 0 \\
\hline \multicolumn{7}{|l|}{ Polychaeta } \\
\hline Opheliidae & 0 & 2200 & 200 & 50 & 400 & 50 \\
\hline Glyceridae & 0 & 50 & 0 & 0 & 50 & 50 \\
\hline Sipunculida & 0 & 50 & 0 & 0 & 50 & 50 \\
\hline Spionidae & 0 & 0 & 0 & 100 & 0 & 1250 \\
\hline Echiuridae & 50 & 0 & 150 & 0 & 50 & 50 \\
\hline \multicolumn{7}{|l|}{ Crustacea } \\
\hline \multicolumn{7}{|l|}{ Hippidae } \\
\hline Emerita rathbunae & 0 & 0 & 0 & 0 & 100 & 0 \\
\hline Insecta & 50 & 0 & 0 & 50 & 0 & 0 \\
\hline
\end{tabular}

61

INVESTIGATIO No. 11, noviembre 2018, Edición Especial, pp. 57-66,

ISSN: 1390 - 6399・ISSN-e: 2602 - 8336 
moluscos, la especie dominante es el gastrópodo Olivella semistriata (700 ind $/ \mathrm{m}^{2}$, zona de retención; Figura 3 ).

La Figura 4 muestra que la zona intermareal puede ser divida en dos áreas en cuanto al macrozoobentos evaluado: la zona seca, caracterizada por individuos pertenecientes a los grupos taxonómicos Echiuridae e Insecta, y otra área compuesta por la zona de retención y resurgencia, caracterizada por el resto de los grupos taxonómicos. Se establecieron diferencias significativas entre las zonas evaluadas (PERMANOVA; PseudoF= 3,01; Pperm $=$ 0,006), lo cual complementa el patrón espacial detectado por el diagrama de la Figura 4.

\section{Monitoreo de avifauna}

A lo largo de los $1500 \mathrm{~m}$ de playa arenosa en El Pelado, se registraron cuatro especies de aves: Haematopus

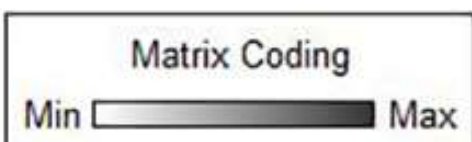

\section{Information Remaining (\%)}

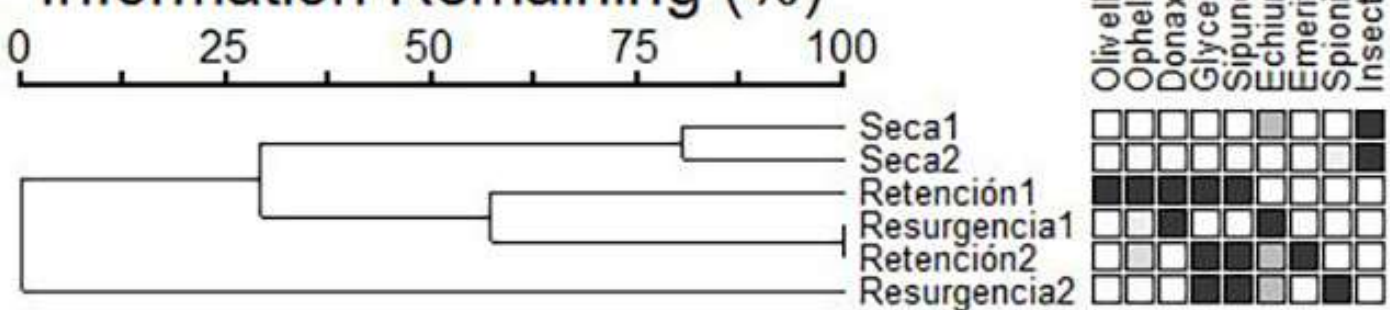

Figura 4. Análisis de Clasificación Jerárquica de doble vía, en donde se muestra cada una de las zonas evaluadas (ver Materiales y Métodos) y los grupos taxonómicos que la caracterizan. Mientras más oscuro es el cuadro, indica una mayor abundancia de ese grupo taxonómico en esa zona. Seca, Retención, y Resurgencia corresponde a las zonas de Salvat (1964), y los números $(1,2)$ indican cada transecto. 
palliatus, Charadrius vociferus, Numenius phaeopus, y Tringa incana. Todas las especies mostraron comportamientos de alimentación, es decir, introdujeron sus picos en la zona intermareal arenosa (Figura 5).

(a)

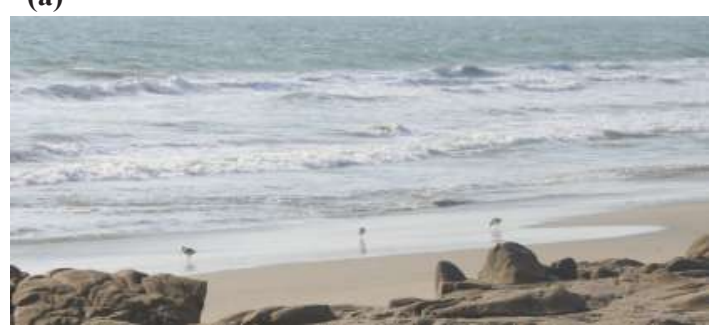

(b)

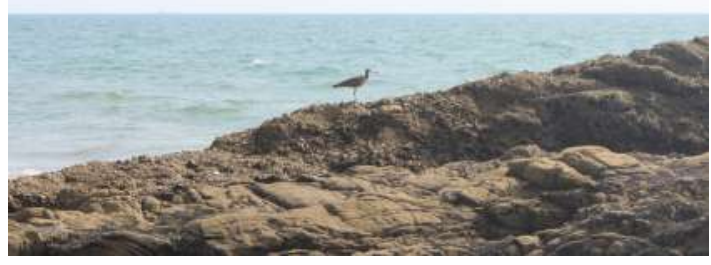

Figura 5. Fotografías de Haematopus palliatus (a) y Numenius phaeopus (b).

\section{Discusión}

En esta evaluación preliminar se muestra que para la playa El Pelado, la composición de especies del macrozoobentos está compuesto principalmente por individuos pertenecientes a cuatro familias de poliquetos, en la cual destaca por su alta densidad la familia Opheliidae, seguido por la familia Spionidae. Otra especie con una densidad alta es el molusco gastrópodo Olivella semistriata. Otro hallazgo destacable es la distribución del macrozoobentos en la zona intermareal, en la cual puede ser dividida en dos zonas en base a las especies que allí habitan. Los individuos de las cuatro especies de aves observadas durante el presente estudio piloto, introdujeron activamente sus picos en el suelo arenoso, lo cual es un indicio comportamental de forrajeo de macrofauna bentónica. Si bien, estas observaciones solo correlacionan la presencia de las especies de aves con lo cual se asume un aprovechamiento de la macrofauna, existe documentación que sustentan que la composición de la dieta de las cuatro especies observadas incluye las especies y varios grupos taxonómicos de macrofauna (Olivella semistriata, Donax mancorensis, Emerita rathbunae, Polychaeta) registrados en este estudio piloto (Hockey et al., 2018; Wiersma et al., 2018; Van Gils et al., 2018 a, b). Esto reafirma la importancia de estudios que relacionen la interacción ecológica entre la macrofauna bentónica de playas arenosas y plataformas rocosas con las actividades de forrajeo de aves playeras residentes y migratorias.

\section{Agradecimientos}

Agradecemos a los estudiantes de pregrado de las asignaturas de Zoología de Invertebrados y Zoología de Vertebrados, I Semestre 2017-2018, de la Carrera de Biología de la Escuela Superior Politécnica del Litoral.

\section{Referencias}

Agreda, A., Villón R., Montenegro B. (2013). Noteworthy bird records from the Santa Elena Peninsula and coastal south-west Ecuador. Cotinga, 35, 99 - 101.

Anderson, M., Gorley, R., Clarke, K. (2008). PERMANOVA + for PRIMER: Guide to Software and Statistical Methods. Plymouth: UK PRIMER-E. 
Aerts, K., Vanagt, T., Degraer S., Guartatanga, S., Wittoeck, J., Fockedey N., Cornejo-Rodriguez, M. P, Calderón, J., Magda Vincx. (2004). Macrofaunal community structure and zonation of an Ecuadorian Sandy beach (bay of Valdivia). Belgian Journal of Zoology, 134, 15-22.

Clarke, K. (1993). Non-parametric multivariate analyses of changes in community structure. Austral Journal of Ecology, 18, 117-143.

Dominguez Granda, L., Fockedey, N., De Mey, M., Beyst B., Cornejo, M., Calderon, J., Vincx, M. (2004). Spatial patterns of the surf zone hyperbenthic fauna of Valdivia Bay (Ecuador). Hidrobiologia, 529, 205-224.

Emery, K. (1961). A simple method of measuring beach profiles. Limnology and Oceanography, 6, 90-93.

Haase B. (2011). Aves marinas de Ecuador continental y acuáticas de las piscinas artificiales de Ecuasal. Guayaquil: Aves \& Conservación, BirdLife International and Ecuasal S.A.

Hernandez, M., Musmeci, L., Bala, L. (2010). Optimización del tiempo de alimentación por parte del playero rojizo (Calidris canutus rufa) en Península Valdes, Patagonia Argentina. Ornitología Neotropical, 21, 445-451.

Herrera, A. (2007). Influencia de los ríos sobre ecosistemas marino- costeros: ejemplos sobre comunidades de playas arenosas. Tesis doctoral. Universidad Simón Bolívar, Venezuela. 238 pp.

Hockey, P., Kirwan, G., Boesman, P. (2018). American Oystercatcher (Haematopus palliatus). En: del Hoyo, J., Elliott, A., Sargatal, J., Christie, D.A. \& de Juana, E. (eds.). Handbook of the Birds of the World Alive. Barcelona: Lynx Edicions.
Disponible en:

https://www.hbw.com/node/53751.

Marin Jarrin, J., Miño Quezada, S., Dominguez Granda, L., Guartatanga Argudo, S., Cornejo, M. (2015). Spatio-temporal variability of the surf-zone fauna of two Ecuadorian Sandy beaches. Marine and Freshwater Research, 66, 1-12.

Marin Jarrin, J., Vanaverbeke, J., Fockedey, N., de Grunauer, M., Domínguez-Granda, L. (2017) Surf zone fauna of Ecuadorian Sandy beaches: Spatial and temporal patterns. Journal of Sea Research, 120 , 41-49.

McCune, B., Grace, J., Urban, D. (2002) Analysis of ecological Communities. $1^{\circ}$ edición. Design. Oregon: M\&M Software.

Musmeci, L., Hernández, M., Scollaro, J., Bala, L. (2013). Almejas en golfos norpatagónicos, Argentina: disponibilidad trófica para aves playeras migratorias. Revista de Biología Marina y Oceanografia, 48, 219-225.

Nuques, V. (2012). Propuesta integral de mejoramiento del turismo de sol y playa como medio efectivo para el incremento de turistas que visitan el Balneario General Villamil de la Provincia del Guayas. Proyecto de titulación previo a la obtención del título de Ingeniero en Administración de Empresas Turísticas y Hoteleras. Universidad Católica de Santiago de Guayaquil, Ecuador, 113 pp.

Pino, M. y Jaramillo, E. (1992). Morphology, texture and mineralogical composition of sandy beaches in the South of Chile. Journal of Coastal Research, 8, 593-602.

Salvat, B. (1964). Les conditions hydrodynamiques interstitielles de sediments meubles intertidaux et la repartition verticale de la faune endogee. Cahier de Recherche l'académie de science, 259, 1576-1579. 
Van Gils, J., Wiersma, P. \& Kirwan, G.M. (2018) a. Wandering Tattler (Tringa incana). En: del Hoyo, J., Elliott, A., Sargatal, J., Christie, D.A. \& de Juana, E. (eds.). Handbook of the Birds of the World Alive. Barcelona: Lynx Edicions. Disponible en https://www.hbw.com/node/53915.

Van Gils, J., Wiersma, P. \& Kirwan, G.M. (2018) b. Whimbrel (Numenius phaeopus). En: del Hoyo, J., Elliott, A., Sargatal, J., Christie, D.A. \& de Juana, E. (eds.). Handbook of the Birds of the World Alive. Barcelona: Lynx Edicions. Disponible en https://www.hbw.com/node/53894.

Vanagt, T. (2007). The role of swash in the ecology of Ecuadorian sandy beach macrofauna, with special reference to the surfing gastropod Olivella semistriata. Tesis Doctoral. Bélgica: Universidad de Gent.

Villamar, F. (2009). Estudio de los poliquetos bentónicos y fauna acompañante en la zona intermareal y submareal de la Bahía de Santa Elena (Ecuador) durante el año 2007. Acta Oceanográfica del Pacífico, 15, 127-138.

Villamar, F. (2013). Estudios de los poliquetos (gusanos marinos) en la zona intermareal y submareal de la bahía de Manta (Ecuador), y su relación con algunos factores ambientales, durante marzo y agosto del 2011. Acta Oceanográfica del Pacífico, 18, 117-130.

Villamar, F., Cruz, M. (2007). Poliquetos y moluscos macrobentónicos de la zona intermareal y submareal en la provincia del Guayas (Monteverde, Ecuador). Acta Oceanográfica del Pacífico, 14, 147-153.

Wiersma, P., Kirwan, G.M. \& Boesman, P. (2018). Killdeer (Charadrius vociferus). En: del Hoyo, J., Elliott, A., Sargatal, J., Christie, D.A. \& de Juana, E. (eds.). Handbook of the Birds of the World Alive. Barcelona: Lynx Edicions, Disponible en https://www.hbw.com/node/53827.
Yan Yang, H., Chen, B., Piersma, T., Zhang, Z., Ding C. (2016). Molluscs of an intertidal soft-sediment area in China: Does overfishing explain a high density but low diversity community that benefits staging shorebirds? Journal of sea Research, 109, 20-28. 
